Check for updates

Cite this: RSC Adv., 2018, 8, 37693

Received 12th October 2018

Accepted 1st November 2018

DOI: $10.1039 / c 8 r a 08465 c$

rsc.li/rsc-advances

\section{Indirect fabrication of versatile 3D microfluidic device by a rotating plate combined 3D printing system $\uparrow$}

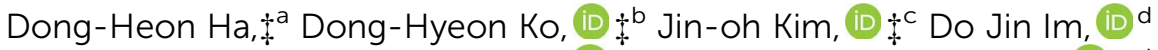 \\ Byoung Soo Kim, ${ }^{a}$ Soo-Young Park, (D) e Steve Park, ${ }^{c}$ Dong-Pyo Kim (iD + tob \\ and Dong-Woo Cho (D) t $^{* a}$
}

In the past decade, 3D-printing technology has been applied in the field of microfluidics to fabricate microfluidic devices for wide-ranging areas of study including chemistry, biology, medicine, and others. However, these methods face several limitations such as insufficient resolution and long fabrication time. In this study, versatile microfluidic devices with different functions were indirectly fabricated by a rapid sacrificial template printing process using a photocurable fluoropolymer with chemical durability. The Pluronic ${ }^{\circ}$ F127 hydrogel as a sacrificial template was rapidly patterned on substrates by a nonlithographic printing process using a computer-controlled 3D-printing system. Viscous fluoropolymer was cast on the non-deformable template that was consequently removed by applying heat and negative pressure after UV curing. The chemical-resistant and transparent microchannels were oblatehemispherical on the cross section. They were tested by performing a heterogeneous catalytic reaction as well as a photochemical reaction. The microchannels with controlled heights were devised to induce convection for functioning as a micromixer with asymmetric flows. Moreover, upon printing the Pluronic $\AA$ F127 on both sides of the PFPE (perfluoropolyether-urethane dimethacrylate) membrane substrate, the 3D hybrid microfluidic device was embedded with a permeable membrane between the lower and upper channels, which is useful for gas-liquid chemical processes.

\section{Introduction}

A microreactor enables chemical reactions to be performed in a confined space several orders of magnitude smaller than conventional reactors. These downsizing effects with a high surface to volume ratio bring a number of attractive features such as fast mixing as well as accurate thermal and reaction time control, and accelerate reaction rates and improve yields.

${ }^{a}$ Department of Mechanical Engineering, Pohang University of Science and Technology (POSTECH), Pohang, South Korea. E-mail: dwcho@postech.ac.kr

${ }^{b}$ Department of Chemical Engineering, Pohang University of Science and Technology (POSTECH), Pohang, South Korea. E-mail: dpkim@postech.ac.kr

'Department of Materials Science and Engineering, Korea Advanced Institute of Science and Technology (KAIST), Daejeon, Korea

${ }^{d}$ Department of Chemical Engineering, Pukyong National University, Busan, South Korea

${ }^{e}$ Department of Polymer Science and Engineering, Kyungpook National University, Daegu, South Korea

$\dagger$ Electronic supplementary information (ESI) available: Movie of the ultra-fast fabrication of microfluidic device and fabrication of dual channel, table of solvent and pressure resistance, figure of controlled chip lengths, ATR spectrum of surface modification steps, ${ }^{1} \mathrm{H}-\mathrm{NMR}$ spectrum Image of PFPE membrane dual channel. See DOI: 10.1039/c8ra08465c

‡ Dong-Heon Ha, Dong-Hyeon Ko, Jin-Oh Kim, Dong-Woo Cho and Dong-Pyo Kim equally contributed to this work.
Moreover, the microfluidic devices can be designed for specific purposes and chemical processes. For example, application of the device to a multi-reaction integrated synthetic process which provides value-added chemicals may improve the process safety by limiting the exposure of the hazardous chemicals to the external environment and shorten the lead time from R\&D to plant construction..$^{1-4}$ In addition, gas-liquid processes in the dual-channel microfluidic systems achieved better diffusion behavior with no formation of random plugs that reduce contact area, when compared to routine single channel microreactors and falling reactors., ${ }^{5,6}$ Therefore, new conceptual microreactors with innovative goals may pose significant potential as 'chemistry finders' that must be repeatedly modified in response to feedback from chemical experiments.

However, existing techniques (e.g., photolithography, soft lithography) generally involve time-consuming and laborintensive steps to fabricate these devices. For example, channel heights are limited in photolithography-based fabrication technique, and photomask should be changed upon modification of the channel design. In the case of direct photolithography, fabrication time is long and the size of the microchannel is limited within the area exposed to $\mathrm{UV}^{{ }^{7-9}}$ Besides, microfluidic devices with multi-dimensional and multifarious microstructures required highly sophisticated 
lithographic techniques such as multi-step lithography or greyscale photolithography. ${ }^{10-13}$

Recently, various printing systems, including 3D printers, have facilitated the fabrication of microfluidic devices with either direct 3D-printing methods using commercially available resins, ${ }^{14,15}$ or indirect sacrificial printing methods using wax ${ }^{13,16}$ and hydrogel. ${ }^{17,18}$ The direct 3D-printing methods have demonstrated to fabricate diverse monolithic systems with sophisticated structural designs. ${ }^{14,15}$ However, the microfluidic channels with mostly millimeters scale exceeding the general size $(c a .500 \mu \mathrm{m})$ were achieved with rough surface morphology using limited amount of resin. On the other hand, the indirect sacrificial printing method has been widely attempted by various heterogeneous combinations of templates and matrix resin on various substrates. ${ }^{17}$ Yet, the indirect sacrificial template printing methods enabled to fabricate microfluidic devices only with simple channel designs mainly for biological uses. ${ }^{18}$ Therefore, rapid on-demand sacrificial printing techniques using suitable combinations of resin and sacrificial materials would be desirable to fabricate versatile and functional microfluidic devices with complex designs and chemical resistance, with no limitations of a special 3D-printing system and resin.

Here, we report a non-lithographic indirect fabrication method for multifarious microfluidic systems with a rapid ondemand sacrificial hydrogel printing (SHP) process and a photocurable fluoropolymer resin with chemical durability. The widths and heights for functional microchannels were simply varied with the printing speeds and heights of a 3D-printing system. The sequential casting of fluoropolymer matrix on the sacrificial hydrogel template and fast photo-curing consolidation step enabled to render the optically transparent microchannels with oblate-hemispherical cross section after neat removal of the template by applying mild vacuum. Moreover, the hybrid fluoropolymer-glass microfluidic device with high resistance to solvents and pressure was fabricated to devise a novel micromixer that induced internal convection flows in two splitting-merging microchannels with asymmetric dimension in height and width. Furthermore, the SHP technique was exploited to fabricate the alternative hybrid microfluidic system for gas-liquid process that was embedded with the gaspermeable membrane between the lower and upper channels. The serpentine and dual-channel microreactor was tested for a gas-liquid model reaction by vertically transporting a reactive $\mathrm{NH} 3$ gas in the lower channel into aqueous $\mathrm{CuSO}_{4}$ liquid in the upper channel.

Taken together, it is believed that this SHP technique is a useful platform approach to fabricate hybrid microfluidic devices by using various combinations of matrix resins and functional substrates for with multifarious purposes.

\section{Materials and methods}

\section{Chemicals and materials}

Perfluoropolyether-urethane dimethacrylate (PFPE, Fluoro MD 700), was purchased from Solvay-solexis and its photoinitiator 2-hydroxy-2-methyl-1-phenyl-propan-1-one (Darocur 1173) was purchased from BASF. Pluronic® F127 (F127) hydrogel (30 wt\%, mixed with distilled water at $0{ }^{\circ} \mathrm{C}$ for $48 \mathrm{~h}$ ) was used for sacrificial template. All chemicals and solvents were purchased from Sigma-Aldrich and used without further purification.

\section{Rotating plate combined 3D-printing system (RPPS)}

RPPS, which is one-of-a-kind custom 3D-printing system, moves in the $x$ and $y$ directions, with two heads moving in the $z$ direction (Fig. 1a). Each head is equipped with a controllable heating unit (TCU-02, Musashi engineering Inc.) and a dispenser (ML-5000XII, Musashi engineering Inc.) providing tuneable pneumatic pressure up to $700 \mathrm{kPa}$, so that the molten material can be extruded through the nozzle (Fig. 1b). The system (HX2.0-MC, TURBO CNC Inc.) operates with G-code. A $200 \mathrm{~mm} \times 200 \mathrm{~mm}$ workspace is used for making a channel by printing on a substrate such as glass slides. RPPS has a rotating motor which also functions as the rotating axis. In this experiment, a rotating motor was connected to the rotating plate (printing area: $25 \times 100 \mathrm{~mm}$ ) to which the membrane was loaded and fixed in the planar direction to enable the printing on both sides (Fig. 1c).

\section{Indirect fabrication of microfluidic system by sacrificial template printing process}

In general, a slide glass (S9213, $76 \mathrm{~mm} \times 52 \mathrm{~mm}$ ) was used as a substrate of the microfluidic device. In order to promote the adhesion between the glass and the PFPE fluoropolymer, the glass was chemically activated by applying plasma electrolytic oxidation to form hydroxyl groups. Then a $30 \mathrm{wt} \%$ solution of 3-
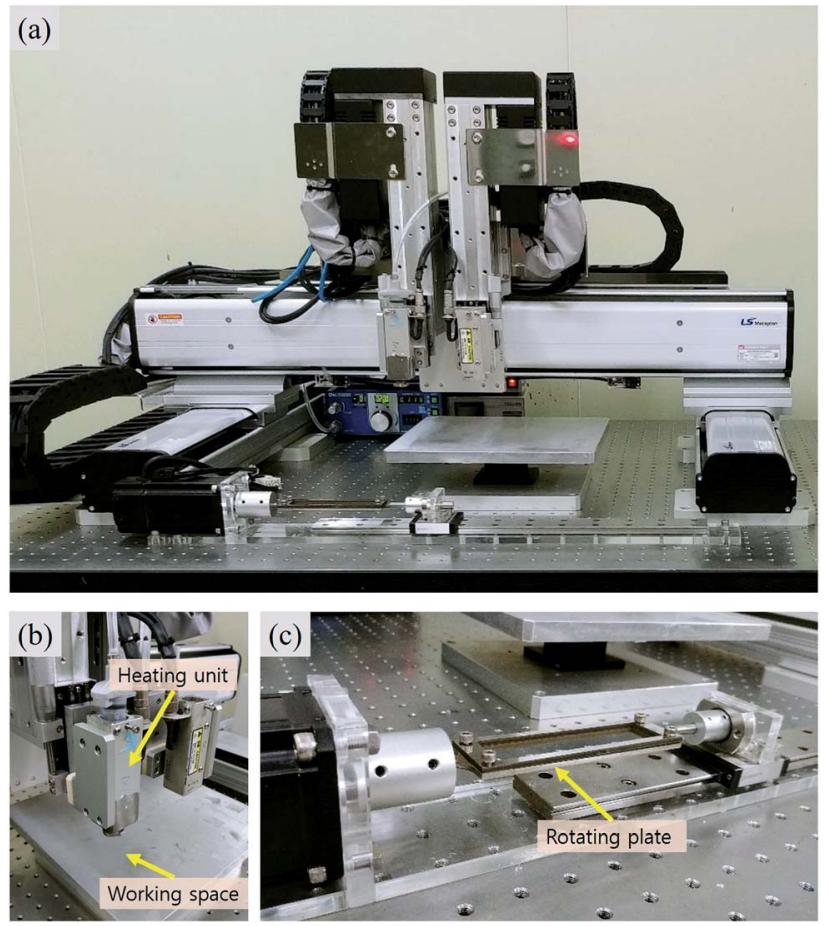

Fig. 1 Rotating Plate combined 3D Printing System (RPPS) for printing microfluidic device. (a) A front view of RPPS, (b) heating unit and working space, (c) rotating plate. 
(a)

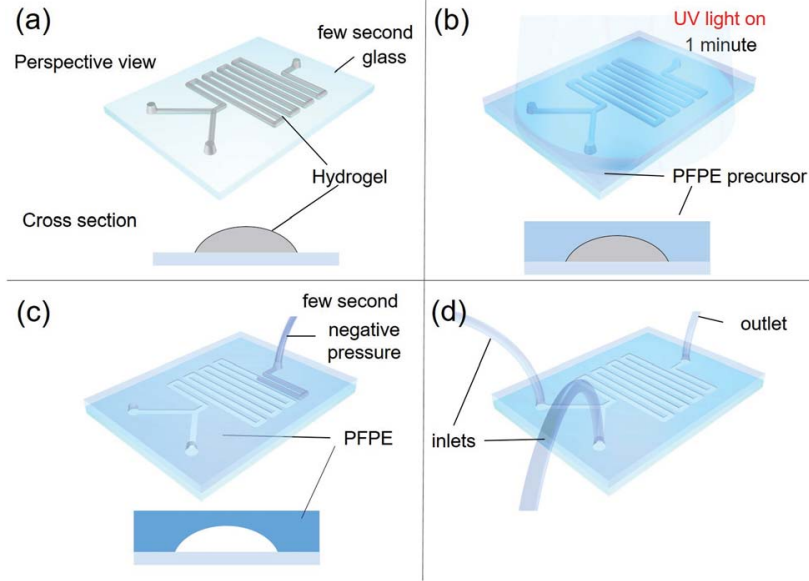

Fig. 2 Scheme for fabricating a microfluidic system. (a) Sacrificial hydrogel printing (SHP) process on MPTMS-treated glass using a 3D printing system. (b) Casting fluoropolymer and photo-consolidation. (c) Removing hydrogel under vacuum pumping. (d) Tubing connection to inlets and outlet.

trimethoxysilylpropyl methacrylate (MPTMS) in toluene was spin-coated at $3000 \mathrm{rpm}$ for $30 \mathrm{~s}$, annealed at $80^{\circ} \mathrm{C}$ for $3 \mathrm{~h}$, and washed with toluene to functionalize the glass surface with acrylate group that reacts with acrylate group of PFPE resin, as previously reported. ${ }^{19}$

Sacrificial F127 was printed through a heated head at $50{ }^{\circ} \mathrm{C}$ on the functionalized glass substrates at room temperature (Fig. 2a; ESI S1 $\dagger$ ). ${ }^{20}$ The printed F127 patterns in height $100 \mu \mathrm{m}$ (can be stacked up to several millimeters) and widths 100, 200, 300,400 and $500 \mu \mathrm{m}$ on glass were covered with $1 \mathrm{~mm}$ thickness of UV-curable PFPE precursor by spin-coating at $300 \mathrm{rpm}$ for $10 \mathrm{~s}$. Then the PFPE precursor was solidified by UV-irradiation for 1 min in $\mathrm{N}_{2}$ atmosphere (Fig. 2b). Inlet and outlet holes were made using a puncher (Uni-core $1.2 \mathrm{~mm}$, Harris) at each end of PFPE/F127 composite patterns, and the F127 patterns was re-annealed at $0{ }^{\circ} \mathrm{C}$ for facile removal by employing vacuum suction (GLD-210B, ULVAC KIKO Inc.) through the holes (Fig. 2c and d), followed by washing the microchannel with pure water.

\section{Preliminary test of the fabricated microfluidic devices}

Solvent resistance was tested by comparing the weights of photo-cured PFPE and polydimethylsiloxane (PDMS) blocks $(10 \mathrm{~mm} \times 10 \mathrm{~mm} \times 1 \mathrm{~mm})$ between the as-prepared and the immersed in various solvents for $24 \mathrm{~h}$, as previously reported. ${ }^{21}$ The pressure resistance of the PFPE and PDMS microfluidic devices (linear channel, length: $5 \mathrm{~cm}$, height: $100 \mu \mathrm{m}$, widths: $100,200,300,400$ and $500 \mu \mathrm{m}$ ) were tested by measuring the burst pressures when a High-Performance Liquid Chromatography (HPLC) pump connected to an inlet and the outlet was blocked. ${ }^{21}$ Water was injected into the microchannel with an increasing flow rate in the range of 0.1 to $10 \mathrm{~mL} \min ^{-1}$ until leakage was observed. At that moment, the maximum pressure was determined as a measure of pressure resistance.

For Suzuki coupling reaction, a flow was given to the microchannel in the device (single linear microchannel, width: $600 \mu \mathrm{m}$, height: $130 \mu \mathrm{m}$, length: $20 \mathrm{~cm}$ ) using Piranha solution
$\left(\mathrm{H}_{2} \mathrm{SO}_{4}: \mathrm{H}_{2} \mathrm{O}_{2}=5: 1, \mathrm{v} / \mathrm{v}\right)$ for a complete removal of $\mathrm{F} 127$ residues as well as to form hydroxyl groups on the glass substrate in the microchannel. Note that the PFPE part was not affected due to its high solvent resistance. For immobilization of metal catalyst, $30 \mathrm{wt} \%$ 3-aminopropyl triethoxysilane (APTES) in ethanol solution was passed through the microchannel to load Pd catalyst by serially infusing $10 \mathrm{mg}$ Pd(II) acetate $\left(\mathrm{Pd}(\mathrm{OAc})_{2}\right)$ in $10 \mathrm{~mL}$ ethanol solution. ${ }^{22,23}$ For the $\mathrm{C}-\mathrm{C}$ coupling reaction, bromobenzonitrile $(0.5 \mathrm{mmol})$ and phenylboronic acid $(0.75 \mathrm{mmol})$ in a mixture of ethanol $(3 \mathrm{~mL})$ and distilled water $(3 \mathrm{~mL})$ solution was prepared, then potassium carbonate $(1 \mathrm{mmol})$ was added. The mixed solution was infused at flow rate $3.3 \mu \mathrm{L} \mathrm{min}{ }^{-1}$ into the Pd catalyst immobilized microchannel devices through single inlet using a syringe pump to yield a product. Secondly, the photochemical C-N coupling reaction with visible light was performed with a $30 \mathrm{~W}$ LED lamp placed $30 \mathrm{~cm}$ above the transparent fluoropolymer-glass microchannel (single linear microchannel, width: $600 \mu \mathrm{m}$, height: $130 \mu \mathrm{m}$, length: $20 \mathrm{~cm}$ ). Note that both PFPE and glass have high transmittance to visible light. ${ }^{21} \mathrm{~A}$ mixture of $(2 E, 7 E)$ 1,9-diphenylnona-2,7-diene-1,9-dione 2-aminophenol ( $0.1 \mathrm{M})$ as a starting reagent, tris(2,2'-bipyridyl) dichlororuthenium(II) hexahydrate (5 mol\%), lithium tetrafluoroborate (2 equiv.) and $\mathrm{N}, \mathrm{N}$-diisopropylethylamine (2 equiv.) dissolved in acetonitrile was injected at flow rate $1.65 \mu \mathrm{L} \mathrm{min}^{-1}$ into the microfluidic device. The collected product was immediately quenched in $1 \mathrm{~mL}$ of distilled water, then the desired products in crude solution were extracted with ether, and dried using a Rotavapor (R-210, BUCHI). The conversions of both reactions were measured by ${ }^{1} \mathrm{H}$ NMR measurements ( $\left.300 \mathrm{MHz}, \mathrm{CDCl}_{3}, \mathrm{JEOL}\right)$.

\section{Splitting-merging type of micromixer fabrication with asymmetric flow}

A micromixer system with total length $40 \mathrm{~cm}$ was composed of 9 sets of splitting and merging sub-channels, which induced asymmetric flow (two inlets $21.2 \mathrm{~mm}$, larger main line 267.2 $\mathrm{mm}$, and 8 smaller lines $109.3 \mathrm{~mm}$ ). Sacrificial hydrogel template patterns consisting of two different widths and heights were individually printed using a 3D-printing system on the MPTMS-coated glass by adjusting printing speed. In the pattern for each sub-channel, the dimension of $500 \mu \mathrm{m}$ wide and $170 \mu \mathrm{m}$ high was made by $150 \mathrm{~mm} \mathrm{~min}^{-1}$ of printing speed, while the dimension of $200 \mu \mathrm{m}$ wide and $50 \mathrm{~mm}$ high was obtained by $500 \mathrm{~mm} \mathrm{~min}^{-1}$ of printing speed. Eventually, a large microchannel of $500 \mu \mathrm{m}$ width and $170 \mu \mathrm{m}$ height (included $90^{\circ}$ angle between $5 \mathrm{~mm}$ and $10 \mathrm{~mm}$ from splitting and merging point, respectively) and small microchannel of $200 \mu \mathrm{m}$ width and $50 \mu \mathrm{m}$ height (included a $135^{\circ}$ angle between 5 and $7.1 \mathrm{~mm}$ from splitting and merging point, respectively) were mutually split and merged along 8 cycles, which were initiated through two inlet streams $(10.6 \mathrm{~mm}$ length for each, $500 \mu \mathrm{m}$ width and $170 \mu \mathrm{m}$ height). In addition, the fabrication conditions such as fluoropolymer casting, photo-curing, and template removal were identical as aforementioned. The mixing efficiency was evaluated by flowing two immiscible solutions of methanol $(50 \%, \mathrm{v} / \mathrm{v})$ and $n$-heptane at flow rates at ranges $40 \mu \mathrm{L} \mathrm{min}{ }^{-1}$ to 
$200 \mu \mathrm{L} \min ^{-1}$. The transferred concentration of Nile red dye initially dissolved only in methanol to $n$-heptane was quantified using a UV-Vis transmittance spectrum at wavelengths from 380 to $780 \mathrm{~nm}^{24}$ For comparison, a Y-shaped and serpentine microchannel with a rectangular cross section $500 \mu \mathrm{m}$ wide, 170 $\mu \mathrm{m}$ high and $234.5 \mathrm{~mm}$ long was fabricated on glass by conventional photo-lithography technique and fluoropolymer resin, but similarly designed with the micromixer in cross sectional area and inner volume.

\section{Membrane embedded dual-channel microreactor}

Polytetrafluoroethylene (PTFE) membrane filter paper (pore size: $0.5 \mu \mathrm{m}$, diameter: $47 \mathrm{~mm}$, Whatman) was used as a printing substrate with moderate flatness. $30 \mathrm{wt} \%$ F127 hydrogel was printed by $400 \mathrm{~mm} \mathrm{~min}^{-1}$ of printing speed to make a serpentine pattern $(300 \mu \mathrm{m}$ wide, $110 \mu \mathrm{m}$ high and $18 \mathrm{~cm}$ long). Then, the mirrored template pattern was printed on the opposite side of the membrane after careful alignment with aid of mark on side of the membrane and printing stage, followed by identical casting and photo-curing of fluoropolymer and template removal as mentioned previously. Eventually, a dual-channel microfluidic system embedded with PTFE membrane was used as a gas-liquid microreactor by reacting a 10 wt $\% \mathrm{CuSO}_{4}$ aqueous solution with $\mathrm{NH}_{3}$ gas. The $\mathrm{NH}_{3}$ gas was generated by heating a $28-30 \% \mathrm{NH}_{3}$ aqueous solution at $40{ }^{\circ} \mathrm{C}$. Residence time was adjusted to $10,20,30$ and $40 \mathrm{~s}$ by controlling flow rates. Unreacted $\mathrm{NH}_{3}$ gas was quenched by collecting with water. UV-Vis absorbance spectrum of product at wavelength $600 \mathrm{~nm}$ (yellow region) was measured at different residence time to monitor the gas-liquid reaction.

\section{Results and discussion}

\section{Rapid sacrificial template printing process}

The printing speed, nozzle size and extrusion pressure is an important factor to control the resulting channel widths. In general, the higher printing speed produced the narrower width of microchannels. The $27 \mathrm{G}$ nozzle with $200 \mathrm{kPa}$ formed $120-600$ $\mu \mathrm{m}$ width patterns at $1000-50 \mathrm{~mm} \mathrm{~min}^{-1}$ of printing speed, respectively. The $27 \mathrm{G}$ nozzle with $400 \mathrm{kPa}$ gave $260-980 \mu \mathrm{m}$ width patterns at $1000-50 \mathrm{~mm} \mathrm{~min}^{-1}$ of printing speed, respectively (Fig. 3).

In addition, the pattern height can be adjusted by controlling the printing height, a distance between the nozzle and the printing surface. It indicates that this SHP technique allowed reproducible production of microchannels with specified height as well as width. For example, the microchannels with a width of $600 \mu \mathrm{m}$ and a height of $130 \mu \mathrm{m}$ were made by printing at $300 \mathrm{~mm} \mathrm{~min}^{-1}$ using $30 \mathrm{wt} \% \mathrm{~F} 127$ hydrogel. Note that there was no observable difference in dimensions between the obtained microchannel and the F127 pattern (width and height).

Eventually, the SHP process enabled successful fabrication of Y-shaped microchannels consistent with the initial CAD design as shown in Fig. $4 \mathrm{a}$ and b. Thus, these microchannels are well ready to use for chemical reactions that require precise control of flow dynamics.

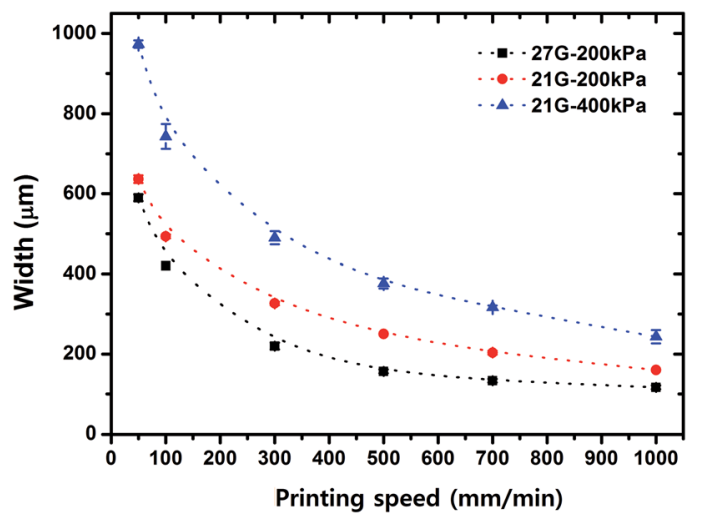

Fig. 3 Relationship between printing speed, extrusion pressure, nozzle size and resulting pattern width of the sacrificial template.

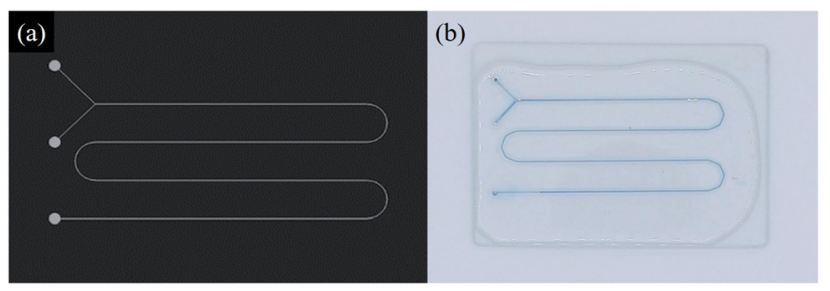

Fig. 4 CAD design and corresponding optical image of the $Y$-shaped microchannel. (width: $600 \mu \mathrm{m}$, height: $130 \mu \mathrm{m}$ ).

\section{Preliminary test of the fabricated microfluidic devices}

As expected, the fluoropolymer PFPE showed superior solvent resistance with a lower swelling ratio in most organic solvents even at $60{ }^{\circ} \mathrm{C}$ to the PDMS. In the pressure resistance tests of hybrid PFPE-glass microfluidic devices (linear length $5 \mathrm{~cm}$ ), the microchannels with various dimensions (height $100 \mu \mathrm{m}$, widths 100-500 $\mu \mathrm{m}$ ), were burst at $670 \mathrm{psi}$, which is much higher than 270 psi of the hybrid PDMS-glass channel. This stronger adhesion is attributed to a chemical bond between the glass surface functionalized with acrylate group and the PFPE resin containing acrylate groups, as similarly reported. ${ }^{19}$ Thus, the hybrid PFPE-glass microchannels are suitable for chemical reactions at high flow rates that endure the induced high pressure on the walls of the microchannel.

To test the effectiveness of the hybrid PFPE-glass microfluidic device as a catalytic microreactor (width: $600 \mu \mathrm{m}$, height: $130 \mu \mathrm{m}$, length: $20 \mathrm{~cm}$ ), two typical chemical reactions such as Suzuki coupling in the presence of immobilized palladium (Pd) catalyst, and photochemical $\mathrm{C}-\mathrm{N}$ coupling under visible light were performed. For Suzuki coupling, the immobilized $\mathrm{Pd}(\mathrm{OAc})_{2}$ catalyst on the amine functionalized glass surface was confirmed by Attenuated Total Reflection (ATR) spectroscopy (Fig. S1 $\dagger$ ). ${ }^{23}$ The hydroxylated surface of glass by Piranha solution was further modified by amine using APTES reagent, where newly appeared a peak at $2900 \mathrm{~cm}^{-1}$ corresponding to the amine group. A reagent mixture of bromobenzonitrile and phenylboronic acid of ethanol solution with potassium carbonate additive flowed into the Pd-immobilized single-line 
serpentine microchannel (Fig. S2a $\dagger$ ). The reaction for $5 \mathrm{~min}$ at room temperature has resulted in $100 \%$ conversion and $99 \%$ yield of 4-phenylbenzonitrile product (Fig. S3†), which is more efficient than the conventional flask reactions taken several hours. ${ }^{25-28}$ For photochemical $\mathrm{C}-\mathrm{N}$ coupling in the optically transparent hybrid PFPE-glass microfluidic device as used for the Suzuki reaction, $100 \%$ conversion and $81 \%$ yield of $(1 R, 5 S, 6 R, 7 S)$-bicyclo[3.2.0]heptane-6,7-diylbis(phenyl-

methanone) product was obtained by $10 \mathrm{~min}$ reaction time at room temperature (Fig. S2b and $\mathrm{S} 4 \dagger$ ), while the conventional bulk reaction produced similar results at extended reaction times. ${ }^{21,29,30}$ These results indicate that the microfluidic device fabricated by the SHP process enabled to perform various organic reactions with high reliability, which is comparable to the capillary microreactors.

\section{Splitting-merging type of micromixer with asymmetric flow}

The micromixer is an important component in microfluidic platforms for complex chemical reactions and biological applications. ${ }^{31}$ In particular, it is well documented that serial lamination during dynamic flows efficiently promoted the mixing efficiency, in which two laminar flows were forced to merge at the horizontal and vertical direction in a serial manner. ${ }^{32-34}$ However, they inevitably required sophisticated fabrication techniques such as multi-level stacking of microstructures. ${ }^{30}$

In this work, a new micromixer system, composed of 8 sets of splitting and merging sub-channels with different heights and widths, was readily fabricated by the SHP process using a 3Dprinting system (Fig. S5a $\dagger$ ). A large microchannel (width: 500 $\mu \mathrm{m}$, height: $170 \mu \mathrm{m}$, degree: $90^{\circ}$ ) and a small microchannel (width: $200 \mu \mathrm{m}$, height: $50 \mu \mathrm{m}$, degree: $48^{\circ}$ ) were continuously split and merged at 8 cycles, which were initiated through two inlet streams and collected at one outlet (Fig. 5a). Following the channel design, each solution must alternatingly flow through the large channel and the small channel in the cyclic diverging and converging manner along $236 \mathrm{~mm}$ in average channel length (total length $40 \mathrm{~cm}$ ) (Fig. S5a $\dagger$ ). This asymmetric microchannel design induced convection flow for functioning as an efficient micromixer. ${ }^{35}$ In particular, when two immiscible liquids of 50 vol\% methanol $\left(0.93 \mathrm{~g} \mathrm{~mL}^{-1}\right)$ and $n$-heptane $(0.68 \mathrm{~g}$ $\mathrm{mL}^{-1}$ ) with a big difference in the density were used as upstream flow, the local convection flow could be enhanced to balance its density difference. The mixing efficiency was determined by measuring the concentration of Nile red dye dissolved in $n$-heptane that was transferred from methanol at various flow rates in range $40-200 \mu \mathrm{L} \mathrm{min-1.} \mathrm{In} \mathrm{order} \mathrm{to} \mathrm{compare} \mathrm{the} \mathrm{mixing}$ efficiency, the conventional Y-shaped serpentine microchannel was fabricated with similar structural parameters $(500 \mu \mathrm{m}$ wide, $170 \mu \mathrm{m}$ high and $234.5 \mathrm{~mm}$ long) except for rectangular cross sectional shape (Fig. S5b $\dagger$ ). Both micromixers have the same inner volume as $14.07 \mu \mathrm{L}$ and same cross sectional area as 0.06 $\mathrm{mm}^{2}$. The maximum UV-Vis absorbance at $498 \mathrm{~nm}$ wavelength was plotted against flow rates (Fig. S5c $\dagger$ and 5b). In general, the mixing efficiency linearly increased with increasing flow rates and reached its maxima at $200 \mu \mathrm{L} \min ^{-1}$. Moreover, the (a)

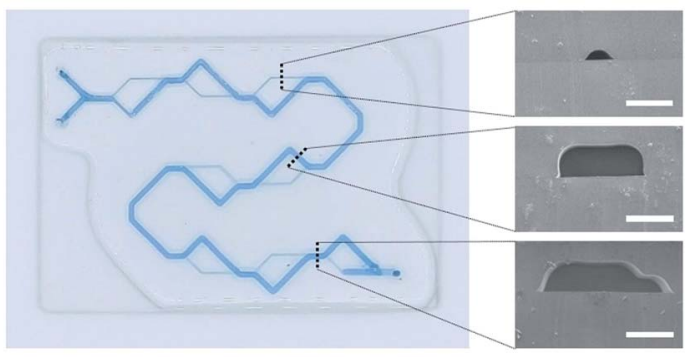

(b)

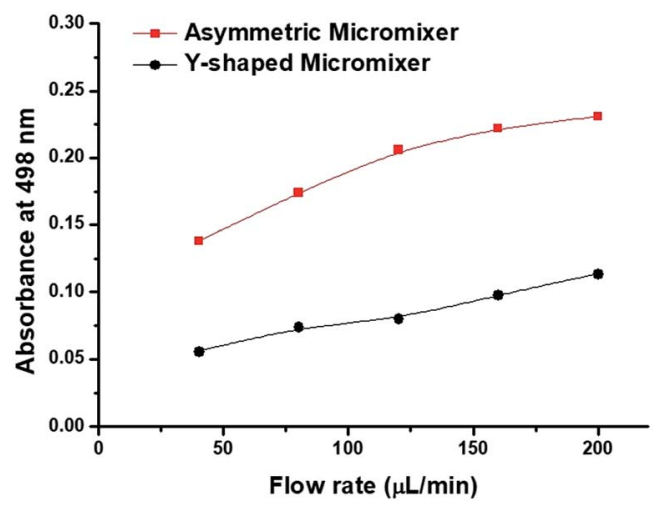

Fig. 5 (a) Optical image and SEM image for an asymmetric splittingmerging type of micromixer (large channel - width: $500 \mu \mathrm{m}$, height: $170 \mu \mathrm{m} / \mathrm{small}$ channel - width: $200 \mu \mathrm{m}$, height: $50 \mu \mathrm{m}$. Scale bar: 250 $\mu \mathrm{m})$. (b) Comparative mixing efficiency between new asymmetric micromixer and ordinary Y-shaped microchannel, measured by UV absorbance $(498 \mathrm{~nm})$ of diffused dye in $n$-heptane.

splitting-merging type of micromixer revealed significantly superior mixing efficiency to the Y-shaped serpentine microchannel at the entire flow rates. The absorbance was about $33.6 \%$ higher for splitting-merging micromixer than those for Yshaped micromixer. Overall, these results demonstrated that the rapid SHP technique is a very useful platform to fabricate an asymmetric flow micromixer with sophisticated design by applying different printing speeds to a 3D-printing system.

\section{Fabrication of 3D dual-channel using rotating plate}

Rotating plate of RPPS is one of the unique component compared with any other commercialized 3D-printing system. Commercialized 3D-printing system only provides an upper surface of bed, but rotating plate enables the printing on both side.

In this study, PTFE membrane was fixed on the rotating plate and sacrificial template was printed in a lattice form (width: 600 $\mu \mathrm{m}$, height: $400 \mu \mathrm{m}$ ) on one side and in helix (line width: 300 $\mu \mathrm{m}$, height: $600 \mu \mathrm{m}$ ) on the other side. The sacrificial template printed PTFE membrane was unloaded from the rotating plate and was filled with PFPE. After crosslinking of PFPE, 3D dualchannel was fabricated (Fig. 6).

\section{Membrane embedded dual-channel microreactor}

Sacrificial template printing can be further applied to fabricate alternative functional microfluidic device embedded with a gas permeable membrane. The serpentine patterns of F127 
(a)

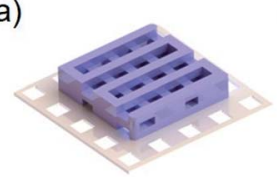

(b)

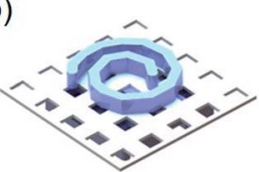

(c)

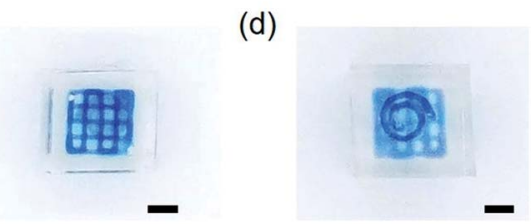

(e)

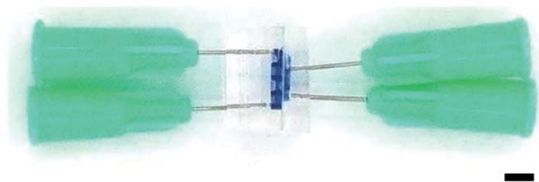

Fig. 6 ( $a$ and b) Upper and bottom CAD image of 3D dual-channel. (c-e) top, bottom, side view of 3D dual-channel (scale bar: $2.5 \mathrm{~mm}$ ).

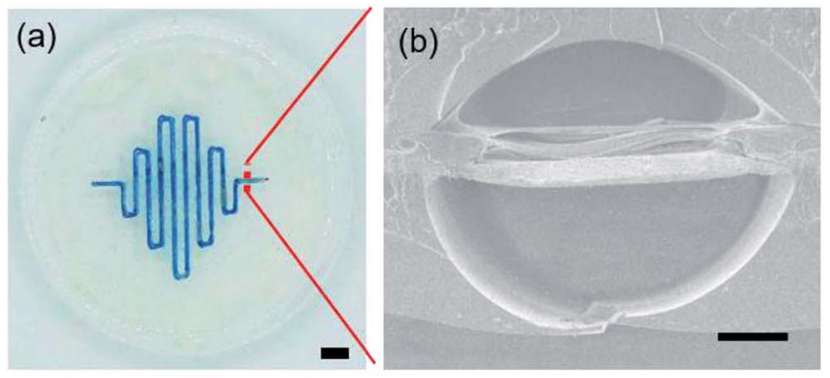

(c)
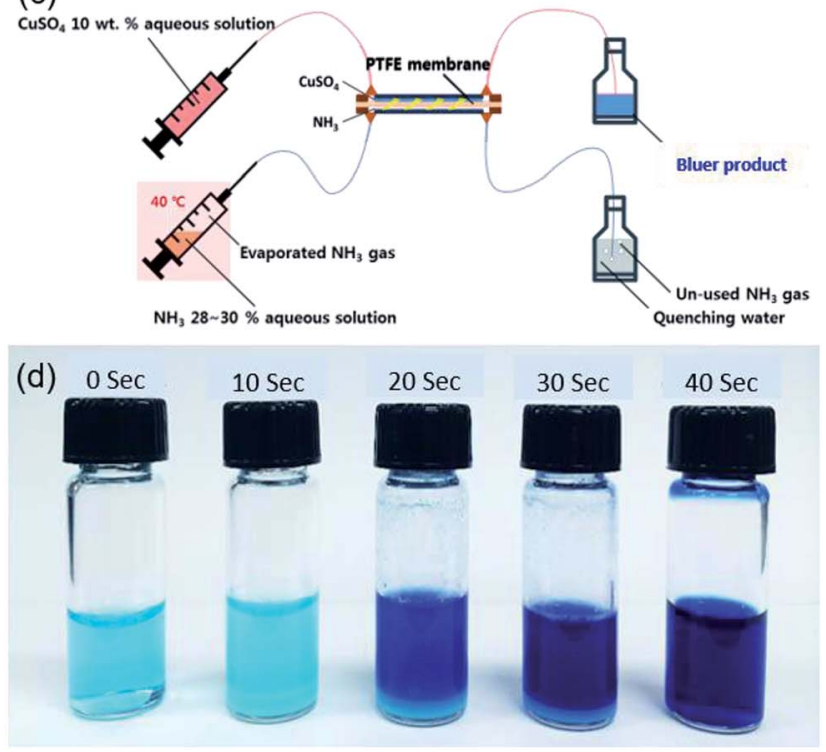

Fig. 7 (a) Top view of PTFE membrane-embedded dual-channel, filled with blue dye (width: $500 \mu \mathrm{m}$, height: $170 \mu \mathrm{m} / \mathrm{scale}$ bar: $5 \mathrm{~mm}$ ). (b) SEM image of the dual-channel cross-section (scale bar: $100 \mu \mathrm{m}$ ). (c) Scheme of $\mathrm{NH}_{3}$ gas $-\mathrm{CuSO}_{4}$ liquid reaction in a dual-channel microreactor. (d) Optical image of products with various residence times. template were printed on both sides of the PTFE membrane substrate after aforementioned careful alignment (ESI Movie $\mathrm{S} 2 \dagger)$. In this step, the hydrophilic F127 could not infiltrate the hydrophobic PTFE membrane pores, where remained white colour due to light scattering effect (Fig. S6a $\dagger$ ). However, it was differently observed that the hydrophobic PFPE resin could readily fill the PTFE membrane pores and became optically transparent (Fig. S6a-c $\dagger$ ). The PFPE filling the gap between the channels is very crucial to block the unwanted lateral diffusion and cross-leakage of gas and liquid reagents across the parallel microchannels. Therefore, the constructed serpentine dualchannel (width: $400 \mu \mathrm{m}$, height: $300 \mu \mathrm{m}$, internal lateral angle: $120^{\circ}$, length: $18 \mathrm{~cm}$ ) on the embedded PTFE filter membrane could allow vertical permeation of gas and hydrophobic liquids across the membrane (Fig. 7a and b).

To demonstrate the feasibility of a PTFE membraneembedded dual-channel as a microreactor for gas-liquid reaction, a $10 \mathrm{wt} \% \mathrm{CuSO}_{4}$ aqueous solution was reacted with $\mathrm{NH} 3$ gas diffused through PTFE membrane from opposite channel (Fig. 7c). As the initial $\mathrm{CuSO}_{4}$ was successfully converted to $\left[\mathrm{Cu}\left(\mathrm{NH}_{3}\right)_{4}\left(\mathrm{H}_{2} \mathrm{O}\right)_{n}\right] \mathrm{SO}_{4}$ complex by ligand change, the solution became deep blue from pale blue (Fig. 7d). ${ }^{36} \mathrm{UV}$-Vis absorbance spectrum at $600 \mathrm{~nm}$ wavelength increased with increasing residence time (Fig. S6d $\dagger$ ). The increase in yellow absorbance indicated the increase of blueness due to the complementary color, consistent with the reaction performance, as expected. Note that the printing capability of various patterns on alternative moderately flat substrates enables to devise functional microfluidic systems.

\section{Conclusions}

A non-lithographic indirect fabrication method using a Rotating Plate combined 3D-printing System for sacrificial template printing has been demonstrated to fabricate multifarious and resistant 3D microfluidic systems, followed by casting and photo-curing of fluoropolymer and template removal steps. The low-cost production process takes only a few minutes, which is far less than alternative fabrication methods. These hybrid fluoropolymer-glass microfluidic chips were designed to perform versatile functions as catalyst-immobilized and photochemical organic microreactors, an asymmetric micromixer and a PTFE membrane embedded dual-channel microreactor for gas-liquid reaction. In particular, a novel asymmetric splitting-merging micromixer with internal convection flows was devised by varying channel dimensions using 3D-printing technology. We have also shown a successful indirect fabrication of $3 \mathrm{D}$ dual-channel using rotating plate. Furthermore, the membrane embedded dual-channel fabricated for gas-liquid reaction was successfully used for vertical diffusion of reactive $\mathrm{NH}_{3}$ gas into the $\mathrm{CuSO}_{4}$ liquid channel with no cross-leakage between paralleled channels. Therefore, it is plausible that this sacrificial hydrogel template printing method may be competitive to 3D-printing technologies as well as lithographic fabrication techniques. As the channel of the microfluidic system fabricated through this technology has a different cross-section dimension compared from the 
conventional channel, there lies a possibility of the difference in fluid flow from the existing one as well. Still, the extension to 3D-printing technology provides synergic opportunities to microfluidics with complex designs and also has numerous potential applications in other fields.

\section{Conflicts of interest}

There are no conflicts to declare.

\section{Acknowledgements}

This study was supported by the National Research Foundation of Korea (NRF) grant funded by the Korean government (MSIP) (no. 2010-0018294) and (no. 2017R1A3B1023598).

\section{Notes and references}

1 R. A. Maurya, C. P. Park, J. H. Lee and D. P. Kim, Angew. Chem., Int. Ed., 2011, 50, 5952-5955.

2 K. C. Basavaraju, S. Sharma, R. A. Maurya and D. P. Kim, Angew. Chem., Int. Ed., 2013, 52, 6735-6738.

3 S. Sharma, R. A. Maurya, K. I. Min, G. Y. Jeong and D. P. Kim, Angew. Chem., Int. Ed., 2013, 52, 7564-7568.

4 K. S. Elvira, X. C. I. Solvas, R. C. R. Wootton and A. J. deMello, Nat. Chem., 2013, 5, 905-915.

5 C. P. Park, R. A. Maurya, J. H. Lee and D. P. Kim, Lab Chip, 2011, 11, 1941-1945.

6 W. Li, K. Liu, R. Simms, J. Greener, D. Jagadeesan, S. Pinto, A. Gunther and E. Kumacheva, J. Am. Chem. Soc., 2012, 134, 3127-3132.

7 J. P. Rolland, R. M Van Dam, D. A. Schorzman, S. R. Quake and J. M. DeSimone, J. Am. Chem. Soc., 2004, 126, 2322-2323.

8 A. Vitale, M. Quaglio, S. L. Marasso, A. Chiodoni, M. Cocuzza and R. Bongiovanni, Direct Photolithography of Perfluoropolyethers for Solvent-Resistant Microfluidics, Langmuir, 2013, 29, 15711-15718.

9 A. Vitale, M. Quaglio, A. Chiodoni, K. Bejtka, M. Cocuzza, C. F. Pirri, et al., Oxygen-Inhibition Lithography for the Fabrication of Multipolymeric Structures, Adv. Mater., 2015, 27, 4560-4565.

10 C. C. Chen, D. Hirdes and A. Folch, Proc. Natl. Acad. Sci. U. S. A., 2003, 100, 1499-1504.

11 C. M. Waits, B. Morgan, M. Kastantin and R. Ghodssi, Sens. Actuators, A, 2005, 119, 245-253.

12 K. Totsu, K. Fujishiro, S. Tanaka and M. Esashi, Sens. Actuators, A, 2006, 130, 387-392.

13 V. Dragone, V. Sans, M. H. Rosnes, P. J. Kitson and L. Cronin, Beilstein J. Org. Chem., 2013, 9, 951-959.

14 K. B. Anderson, S. Y. Lockwood, R. S. Martin and D. M. Spence, Anal. Chem., 2013, 85, 5622-5626.
15 D. Therriault, S. R. White and J. A. Lewis, Nat. Mater., 2003, 2, 265-271.

16 C. J. Hansen, W. Wu, K. S. Toohey, N. R. Sottos, S. R. White and J. A. Lewis, Adv. Mater., 2009, 21, 4143-4147.

17 L. E. Bertassoni, M. Cecconi, V. Manoharan, M. Nikkhah, J. Hjortnaes, A. L. Cristino, G. Barabaschi, D. Demarchi, M. R. Dokmeci, Y. Z. Yang and A. Khademhosseini, Lab Chip, 2014, 14, 2202-2211.

18 D. B. Kolesky, R. L. Truby, A. S. Gladman, T. A. Busbee, K. A. Homan and J. A. Lewis, Adv. Mater., 2014, 26, 31243130.

19 A. Vitale, M. Quaglio, M. Cocuzza, C. F. Pirri and R. Bongiovanni, Eur. Polym. J., 2012, 48, 1118-1126.

20 J. Y. Kim, E. K. Park, S. Y. Kim, J. W. Shin and D. W. Cho, J. Micromech. Microeng., 2009, 19, 104003.

21 J. O. Kim, H. Kim, D. H. Ko, K. I. Min, J. Im do, S. Y. Park and D. P. Kim, Lab Chip, 2014, 14, 4270-4276.

22 Z. Y. Xiao, Y. Zhao, A. J. Wang, J. Perumal and D. P. Kim, Lab Chip, 2011, 11, 57-62.

23 X. D. Le, Z. P. Dong, Y. S. Liu, Z. C. Jin, T. D. Huy, M. Le and J. T. Ma, J. Mater. Chem. A, 2014, 2, 19696-19706.

24 S. Panic, S. Loebbecke, T. Tuercke, J. Antes and D. Boskovic, Chem. Eng. J., 2004, 101, 409-419.

25 Z. G. Zhou, M. Y. Liu, X. L. Wu, H. W. Yu, G. H. Xu and Y. R. Xie, Appl. Organomet. Chem., 2013, 27, 562-569.

26 A. Kumar, G. K. Rao, F. Saleem, R. Kumar and A. K. Singh, J. Hazard. Mater., 2014, 269, 9-17.

27 N. Fukaya, M. Ueda, S. Onozawa, K. K. Bando, T. Miyaji, Y. Takagi, T. Sakakura and H. Yasuda, J. Mol. Catal. A: Chem., 2011, 342-43, 58-66.

28 P. H. Li, L. Wang, L. Zhang and G. W. Wang, Adv. Synth. Catal., 2012, 354, 1307-1318.

29 L. C. Wang, H. Y. Jang, Y. Roh, V. Lynch, A. J. Schultz, X. P. Wang and M. J. Krische, J. Am. Chem. Soc., 2002, 124, 9448-9453.

30 T. G. Baik, A. L. Luis, L. C. Wang and M. J. Krische, J. Am. Chem. Soc., 2001, 123, 6716-6717.

31 N. T. Nguyen and Z. G. Wu, J. Micromech. Microeng., 2005, 15, R1-R16.

32 B. L. Gray, D. Jaeggi, N. J. Mourlas, B. P. van Drieenhuizen, K. R. Williams, N. I. Maluf and G. T. A. Kovacs, Sens. Actuators, A, 1999, 77, 57-65.

33 M. S. Munson and P. Yager, Anal. Chim. Acta, 2004, 507, 6371.

34 N. Schwesinger, T. Frank and H. Wurmus, J. Micromech. Microeng., 1996, 6, 99-102.

35 D. S. Kim, S. H. Lee, T. H. Kwon and C. H. Ahn, Lab Chip, 2005, 5, 739-747.

36 Y. Zhao, M. L. Qin, A. J. Wang and D. Kim, Adv. Mater., 2013, 25, 4561-4565. 